\title{
Electromagnetic Properties of a Plasma Covered Antenna
}

\author{
Daniel J. Jacavanco
}

\author{
Air Force Cambridge Research Laboratories, Bedford, Mass.
}

(Received October 22, 1964; revised February 12, 1965)

\begin{abstract}
Antenna patterns ( $\boldsymbol{E}$-plane) of an X-band horn, mounted in a ground plane and covered by a plasma slab were measured. A pulsed plasma was produced by capacitor discharge in a rectangular Pyrex container of i.d. 6 in. $x 2$ in. $\times 24$ in. Microwave interferometer measurements of the electron density were made at 9 and $35 \mathrm{GHz}$. Antenna patterns are presented which show a sharp decrease in radiated power at certain angles corresponding to the "optics critical angle $\theta_{c}$." Good agreement is obtained for the movement of $\theta_{c}$ with plasma density.
\end{abstract}

\section{Introduction}

The purpose of this paper is to report an experiment to verify certain characteristics of the power pattern of a plasma covered antenna. It is an extension of a project which has been reported in PTGAP [Jacavanco and Meltz, 1964].

The subject of plasma covered antennas has received extensive theoretical treatment. A variety of sources embedded in both magnetostatic and nonmagnetostatic plasmas have been investigated.

However, it has been difficult to show the theoretically predicted antenna patterns experimentally [Flock and Elliot, 1962; Cloutier, Bachynski, and Graf, 1962]. Plasma effects have been masked by container effects. This laboratory conducted a systematic study of various containers and found that the outside edges of the plasma vessel, perpendicular to the plane of the antenna patterns measured, are responsible for perturbing the free space pattern of the antenna. The geometry of this experiment represents a significant improvement over previous attempts, i.e., the maximum ripple in the power pattern is of the order of $2 \mathrm{~dB}$. It will be seen that this experiment has also approached the infinite geometry in that the antenna patterns clearly show the variation of the critical angle with plasma density and display the abrupt discontinuity in the power pattern, characteristic of infinite plasma slabs.

\section{Experiment}

The experiment consists of a rectangular glass container of i.d. 2 in. $x 6$ in. $x 24$ in.; constructed of $3 / 8$ in. Pyrex glass. The edges are ground so as to be interlocking and are sealed with an epoxy resin. An Xband horn, mounted in a ground plane and fed by the $\mathrm{TE}_{10}$ mode is positioned against the $6 \mathrm{in}$. $\mathrm{x} 24 \mathrm{in}$. face with the electric field alined along the long dimension of the bottle (see fig. 1). The horn has an aperture of $\lambda$ by $\lambda$ and is constructed with a gradual taper. The antenna patterns are measured by a 7 in. parabolic receiving antenna which is rotated on a $6 \mathrm{ft}$ arc. Angular position is obtained by a servomechanism. Due to the pulsed nature of the plasma, a $60 \mathrm{deg}$ half plane pattern is taken by positioning the parabolic every 3 deg in the far field and measuring the received power as the plasma decays. An anechoic chamber is constructed of microwave absorber about the experiment to minimize spurious reflections.

The gradual taper of the horn produced a plane wave at the aperture. For this reason it was also used as the transmitting antenna of the microwave interferometer. The interferometer consisted of a waveguide "magic tee" in which a reference signal and a wave which had traversed the plasma were compared. By initially balancing one against the other using a precision phase shifter and attenuator, a time history of the plasma decay was obtained from the unbalanced state of the microwave bridge. In this manner, reproducible densities in excess of $10^{13}$ electrons $\mathrm{cm}^{3}$ were measured at $35 \mathrm{GHz}$. Antenna patterns and density measurements were made at $9 \mathrm{GHz}$.

The plasma is produced by capacitor discharge. A one $\mu \mathrm{F}$ capacitor is charged to $5 \mathrm{kV}$ by a power supply capable of $0.25 \mathrm{~A}$ at $20 \mathrm{kV}$. The capacitor is "crow barred" by a 4C35 hydrogen thyratron twice a second. The $12 \mathrm{~J}$ are discharged into helium at 700 $\mu$ pressure in approximately $10 \mu$ sec with a peak current of $1500 \mathrm{~A}$.

A pulse generator producing two pulses per second is used as the time base. The same pulse which initiates conduction in the hydrogen thyratron also triggers the oscilloscope. By using a dual trace plug-in unit, the transmitted power and the interferometer output are presented simultaneously and photographed. In this manner, the transmitted power level at any angle in the antenna pattern, at any time in the afterglow, is obtained from the photographs of the oscilloscope traces. 


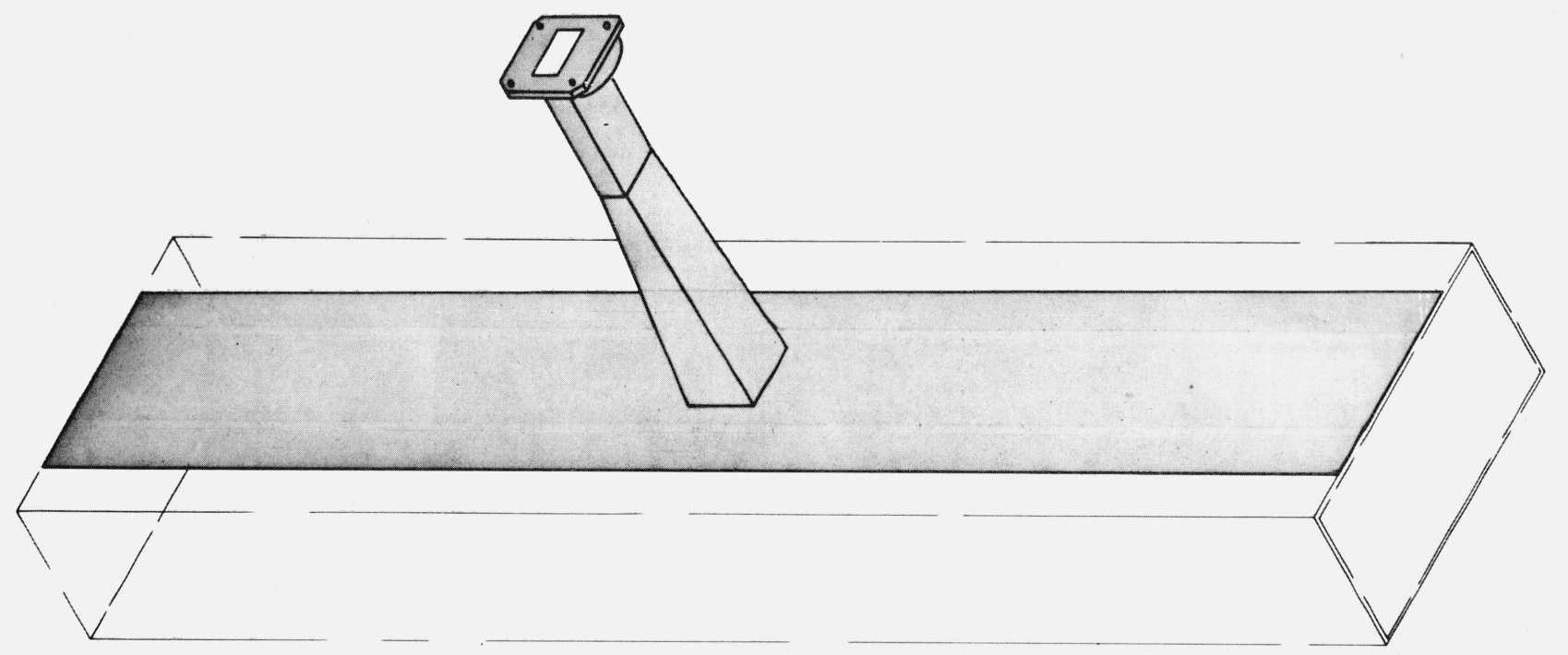

Figure 1. Antenna and plasma container.

Standard vacuum techniques have been utilized, and epoxy resin was used to seal together the plasma container. After heating to $300{ }^{\circ} \mathrm{C}$, leaks developed that were sealed with glyptol. An ultimate vacuum of $8 \times 10^{-7}$ torr was achieved with a hold off vacuum of $10^{-4}$ torr. Since basic microscopic processes were not investigated in this experiment, but rather only the volume effects of electron density upon the spatial power distribution of an antenna, these characteristics of the vacuum were deemed sufficient. This reasoning is justified since reproducible plasmas were obtained.

\section{Theory}

Simply stated, a plasma covered antenna has the following characteristics:

(1) Overall reduction in signal level due to attenuation and reflection,

(2) a peak in the antenna pattern at the critical angle, and angle.

(3) no radiation for angles greater than the critical

The theoretical power pattern is obtained by assuming an aperture distribution, meeting boundary conditions, and evaluating the integral by the saddle point integration techniques.

Insight into the physics of the problem is obtained by considering refraction by a dielectric as described by Snell's law:

$$
\frac{\sin \theta_{1}}{\sin \theta_{2}}=\frac{n_{2}}{n_{1}}
$$

where $\theta_{1}$ is the angle if incidence of the wave (light wave or microwave) in medium one and $\theta_{2}$ is the angle of refraction in medium two. The index of refraction, $n=\sqrt{1-\left(\frac{\omega_{p}}{\omega}\right)^{2}}$ for a collisionless plasma, where $\omega_{p}$ is the plasma frequency and $\omega$ is the frequency of the radiation. If we assume a wave is propagating from the semi-infinite plasma into free space, we obtain the critical angle by letting the angle of incidence in medium one (plasma) be 90 deg. Hence, $\sin \theta_{1}=1$. If medium two is air, $n_{2}=1$. The angle of refraction for this incident angle is the critical angle for the interface and Snell's law becomes

$$
\sin \theta_{c}=n_{1}=\sqrt{1-\left(\frac{\omega_{p}}{\omega}\right)^{2}} .
$$

As the plasma density increases from zero to a value greater than the cutoff density $\left(\frac{\omega_{p}}{\omega}>1\right)$, the critical angle moves from endfire $\theta_{2}=90^{\circ}$ to broadside $\theta_{2}=0^{\circ}$.

\section{Results}

Symmetry was obtained in all antenna patterns, consequently only half plane patterns are presented. Figure 2 shows the comparison between the free space pattern of the antenna with and without the plasma container in place. Here is shown the first criterion for meaningful results: there is no significant difference between the two power patterns, i.e., the container has negligible effect.

Figure 3 is a composite picture of four antenna patterns taken at different densities. The critical angle for each density is also indicated. The abrupt discontinuity in the power pattern occurs at the critical angle and moves toward broadside as the density is increased. The transmitted power level in the broadside direction is also reduced as the density is increased. 


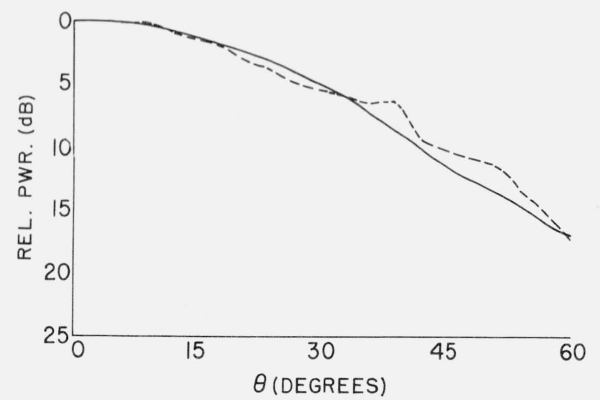

FiguRE 2. Free space antenna patterns showing plasma container perturbation.

\section{Conclusion}

The conclusion reached by this experiment is that although an exact correlation between theory and experiment for the power pattern of a plasma covered antenna is not possible, the physics of the problem can be shown. That is, the abrupt spatial discontinuity in the antenna pattern at the critical angle can be demonstrated and its movement, with plasma density, verified.

The experiment does prove, to some extent, the predictions that Oliner and Tamir expressed in their work on the effects of semi-infinite plasma slabs upon radiation patterns [Oliner and Tamir, 1962]. They have shown that when the distance from the source to the discontinuity, in the plane of the antenna pattern, is greater than $10 \lambda$, the plasma slab appears infinite in extent. The abrupt discontinuity in the antenna patterns presented, and the lack of radiation for angles greater than the critical angle show this. They also predict that when the slab thickness is small there are fewer lobes in the antenna pattern at angles less than the critical angle, and that the peak at the critical angle is reduced. This is also evident in the antenna patterns presented. Hence, good qualitative agreement has been obtained for the pattern of a plasma covered antenna and for the movement of the critical angle with plasma density according to the simple models used.

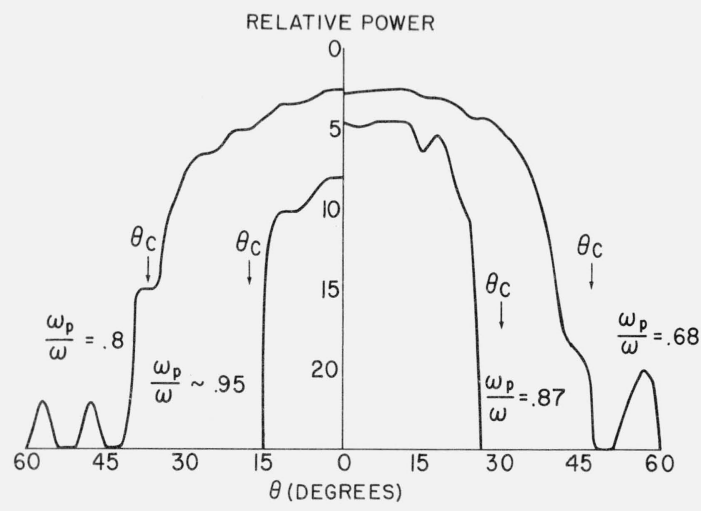

FIGURE 3. Measured patterns of plasma covered antenna at different densities.

The author recognizes the indispensible technical assistance of George Willis. He thanks S. B. Herskovitz and K. V. N. Rao for many helpful discussions and M. J. Cregan for the glass blowing.

\section{References}

Cloutier, G. G., M. P. Bachynski, and K. A. Graf (March 1962), Antenna properties in the presence of ionized media, RCA Victor, Res. Lab. Ltd. Montreal, Canada, AFCRL-62-191.

Flock, W. L., and R. S. Elliot (Jan. 1962), The radiation pattern of a microwave horn and a plasma layer, IRE Trans. Ant. Prop. AP-1 0, 65-68.

Jacavanco, D., and G. Meltz (May 1964), An experimental investigation of antenna pattern distortion due to a plasma layer, IRE Trans. Ant. Prop. AP-1 2, 365-366.

Oliner, A. A., and T. Tamir (May 1962), Radiation from semi-infinite slot-excited plasma sheath configurations, Microwave Research Institute, Polytechnic Institute of Brooklyn, Rpt. No. 1029-62.

(Paper 69D7-530) 